\title{
Multipole and S-parameter Based Antenna Model
}

\author{
Mark Haynes* and Mahta Moghaddam \\ Electrical Engineering and Computer Science \\ University of Michigan, Ann Arbor, MI \\ E-mail: mshaynes@umich.edu, $\underline{\text { mmoghadd@umich.edu }}$
}

\begin{abstract}
Antenna characterization relating voltage sources to radiated fields is an important part of any electromagnetic measurement, with applications ranging from two-way communication systems to full-wave inverse scattering. Here, we derive a two-antenna model relating the multipole expansion of the fields radiated and received by antennas to S-parameter measurements between them. We also show how to determine the antenna model parameters from numerical simulation. Finally, we validate the model experimentally using two rectangular waveguides. This model is based on the sourcescattering matrix used in near-field antenna measurements [2-4], where we have extended it for direct S-parameter measurements.
\end{abstract}

\section{Formulation}

\section{Antenna and S-Parameter Model}

Let the total electric field around an antenna be expressed by the multipole expansion

$$
\boldsymbol{E}(\boldsymbol{r})=\Sigma_{l m}\left[a_{l m} \boldsymbol{M}_{l m}(\boldsymbol{r})+b_{l m} \boldsymbol{N}_{l m}(\boldsymbol{r})+c_{l m} \mathfrak{R} \boldsymbol{M}_{l m}(\boldsymbol{r})+d_{l m} \Re \boldsymbol{N}_{l m}(\boldsymbol{r})\right]
$$

where $\boldsymbol{M}$ and $\boldsymbol{N}$ are the vector spherical harmonics given in [1], and $\Re$ means the regular Bessel function for incoming harmonics. The number of harmonics needed to represent a field produced by an antenna is $O(k d)$, where $d$ is the largest dimension of the antenna, and $k$ is the wavenumber of the medium.

We model the full antenna response following the source-scattering matrix formulation [2-4]. The antenna is connected to a waveguide with source or receiver, shown in Figure 1 , where $a_{o}$ and $b_{o}$ are the complex outgoing and incoming modes on the waveguide, respectively. We relate the waveguide modes to field coefficients through a linear model

$$
\begin{gathered}
b_{o}=a_{o} \Gamma+\Sigma_{l m}\left(u_{(c) l m} c_{l m}+u_{(d) l m} d_{l m}\right) \\
a_{l m}=a_{o} t_{(a) l m}+\Sigma_{l^{\prime} m^{\prime}}\left(S_{(a c) l m, l^{\prime} m^{\prime}} c_{l^{\prime} m^{\prime}}+S_{(a d) l m, l^{\prime} m^{\prime}} d_{l^{\prime} m^{\prime}}\right) \\
b_{l m}=a_{o} t_{(b) l m}+\sum_{l^{\prime} m^{\prime}}\left(S_{(b c) l m, l^{\prime} m^{\prime}} c_{l^{\prime} m^{\prime}}+S_{(b d) l m, l^{\prime} m^{\prime}} d_{l^{\prime} m^{\prime}}\right)
\end{gathered}
$$

where $\Gamma$ is the reflection coefficient looking into the antenna. Quantities $u_{(c) l m}$, and $u_{(d) l m}$ are receive coefficients which convert incoming field harmonics to $b_{o}$. Similarly, $t_{(a) l m}$ and $t_{(b) l m}$ are transmit coefficients which convert $a_{o}$ to outgoing field harmonics. The terms $S_{(x y) l m, l^{\prime} m^{\prime}}$ capture the passive scattering properties of the antenna, similar to a T-matrix. Evoking the reciprocity theorem, with $\boldsymbol{M}$ and $\boldsymbol{N}$ conventions of [1], it can be shown [3] that the transmit and receive coefficients are reciprocally related 


$$
u_{(c, d) l m}=\frac{Z o}{2 k \omega \mu} l(l+1)(-1)^{-m} t_{(a, b) l,-m} .
$$

Next, let two antennas be separated by some distance in free space, shown in Figure 2. One antenna transmits in reference frame $i$ and the other receives in reference frame $j$. Each antenna has its own field expansion and set of transmit and receive coefficients, indexed with $i$ and $j$, respectively. We assume that, 1) there are no incoming waves in the transmitter frame $\left.\left(c_{l m}^{i}=d_{l m}^{i}=0\right), 2\right)$ the receiver is purely receiving $\left(a_{o}^{j}=0\right)$, and 3 ) there is no multiple scattering between the antennas or between antennas and other objects $\left(S_{(x y) l m, l^{\prime} m^{\prime}}=0\right)$. The field coefficients in each frame, $\left(a_{l m}^{i}, b_{l m}^{i}\right.$ and $\left.c_{l m}^{j}, d_{l m}^{j}\right)$ are related through the addition theorem for vector spherical harmonics [1].

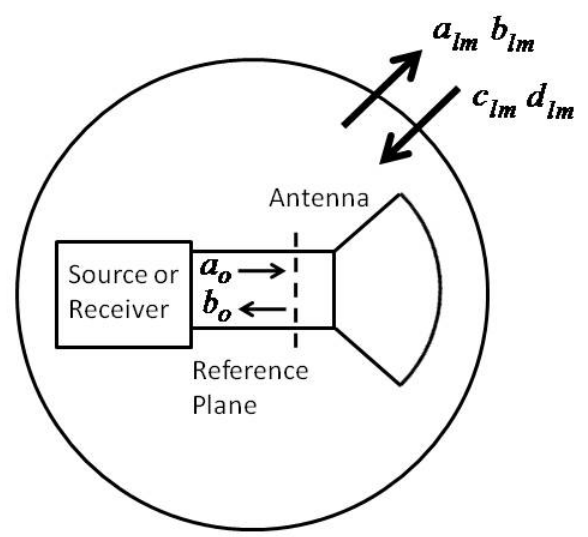

Figure 1: Antenna and waveguide setup

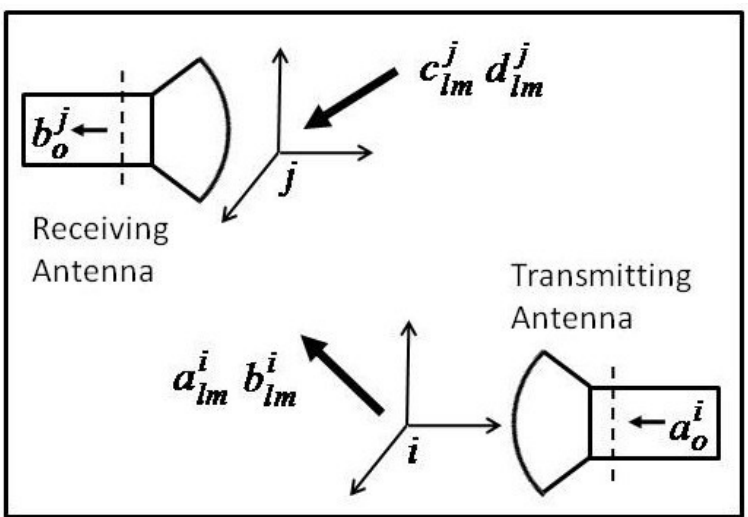

Figure 2: Two antenna setup in free space. Transmitting antenna is in frame $i$; receiving antenna is in frame $j$.

After applying the assumptions above to Eqns. (2), together with the addition theorem, it can be shown that

$$
\begin{aligned}
b_{o}^{j}=a_{o}^{i} \Sigma_{l^{\prime} m^{\prime}} & \left(u_{(c) l^{\prime} m^{\prime}}^{j} \Sigma_{l m}\left(A_{l^{\prime} m^{\prime}, l m}^{j i} t_{(a) l m}^{i}+B_{l^{\prime} m^{\prime}, l m}^{j i} t_{(b) l m}^{i}\right)\right. \\
& \left.+u_{(d) l^{\prime} m^{\prime}}^{j} \Sigma_{l m}\left(B_{l^{\prime} m^{\prime}, l m}^{j i} t_{(a) l m}^{i}+A_{l^{\prime} m^{\prime}, l m}^{j i} t_{(b) l m}^{i}\right)\right)
\end{aligned}
$$

where $A_{l^{\prime} m^{\prime}, l m}^{j i}$, and $B_{l^{\prime} m^{\prime}, l m}^{j i}$ are translation matrices. Finally, using the reciprocity relation for the transmit and receive coefficients, rearranging the sums, and identifying the S-parameter, $S_{j i}=b_{o}^{j} / a_{o}^{i}$, we can write

$$
\begin{gathered}
S_{j i}=\frac{z_{o}}{2 k \omega \mu} \Sigma_{l^{\prime} m^{\prime}} l^{\prime}\left(l^{\prime}+1\right)(-1)^{m^{\prime}}\left(t_{(a) l^{\prime} m^{\prime}}^{j} \Sigma_{l m}\left(A_{l^{\prime},-m^{\prime}, l m}^{j i} t_{(a) l m}^{i}+B_{l^{\prime},-m^{\prime}, l m}^{j i} t_{(b) l m}^{i}\right)\right. \\
\left.+t_{(b) l^{\prime} m^{\prime}}^{j} \Sigma_{l m}\left(B_{l^{\prime},-m^{\prime}, l m}^{j i} t_{(a) l m}^{i}+A_{l^{\prime},-m^{\prime}, l m}^{j i} t_{(b) l m}^{i}\right)\right)
\end{gathered}
$$

where we have formally related the transmit coefficients of each antenna to S-parameter measurements between them. This was possible because we began with an antenna model relating waveguide modes to field harmonics. It can be shown that this relation is reciprocal, $S_{j i}=S_{i j}$. Equation (5) can also be generalized for rotated coordinate systems by inserting rotation matrices before and after the translation matrices. 


\section{Determining Transmit Coefficients from Simulation}

The transmit coefficients, $t_{(a) l m}$ and $t_{(b) l m}$, are also those sought in near field antenna measurements [4]. In the absence of near field measurements, one can determine them approximately from simulation. Unlike experiment, in simulation we can directly sample the field radiated by an antenna and use the approximate numeric field measurements, together with the antenna model, to invert Eqn. (1) for the transmit coefficients.

Let a single antenna be purely radiating, $a_{l m}=a_{o} t_{(a) l m}$ and $b_{l m}=a_{o} t_{(b) l m}$. The electric field in Eqn. (1) is computed at points $\boldsymbol{r}_{n}$, where $n$ indexes spatial samples. We can then write the linear system over all numeric field measurements as

$$
\begin{array}{lll} 
& \boldsymbol{x}=\left[\begin{array}{c}
\boldsymbol{t}_{\boldsymbol{a}} \\
\boldsymbol{t}_{\boldsymbol{b}}
\end{array}\right] \\
\boldsymbol{A} \boldsymbol{x}=\boldsymbol{b} \quad \text { with } \quad[\boldsymbol{b}]_{n}=\boldsymbol{E}\left(\boldsymbol{r}_{n}\right) / a_{o} \\
& & {[\boldsymbol{A}]_{n}=\left[\boldsymbol{M}\left(\boldsymbol{r}_{n}\right) \boldsymbol{N}\left(\boldsymbol{r}_{n}\right)\right]}
\end{array}
$$

where the $l m$ index has been condensed and the field vectors are understood. We determine the transmit coefficients by solving the normal equations of (6) with Tikhonov regularization, $\boldsymbol{x}=\left(\boldsymbol{A}^{*} \boldsymbol{A}+\gamma \boldsymbol{I}\right) \boldsymbol{A}^{*} \boldsymbol{b}$, where ${ }^{*}$ is conjugate transpose.

\section{Experimental Validation}

To validate Eqn. (5) experimentally, we constructed two, hollow, probe-fed brass waveguides with the rationale that these structures could be accurately simulated in Ansoft HFSS. The waveguides were designed with WR-187 specifications to operate between 3.95-5.85 GHz. The probe position was optimized in HFSS for best matching at $5 \mathrm{GHz}$. After construction, we carefully measured the waveguide dimensions and used these dimensions for field simulations, shown in Figure 3. The aperture of the waveguide is the source we wish to model, so the coordinate origin was placed at the center of the aperture. The HFSS waveport of the CAD model was deembedded to the same reference plane of the physical waveguide that would be used by the Network Analyzer.

The value of $S_{11}$ of each physical antenna was measured and compared with simulation. Away from resonance, these were accurate within 5 degrees of phase, and near resonance these were within 30 degrees, confirming that the CAD model was accurate. Returning to simulation, we sampled the fields radiated by the aperture on a sphere with a radius of $20 \mathrm{~cm}$. Using these numeric field measurements, we solved Eqn. (6) for the waveguide transmit coefficients, with harmonics up to $1=10$ and the normalization $a_{o}$ given from simulation. This was done at 21 frequencies between 4-6 GHz.

Having numerically determined the transmit coefficients, the two physical waveguides were attached to a $1 \mathrm{~m}$ Styrofoam board separated by approximately $0.5 \mathrm{~m}$. The value of $S_{21}$ was measured from 4-6 GHz in an anechoic chamber for various distances and rotation angles. Figure 4 shows a case where the waveguide apertures were separated by $0.55 \mathrm{~m}$ and each waveguide was rotated 30 degrees. Eqn. (5) was then evaluated for the same distance and rotations with the computed transmit coefficients. Figures 5 and 6 are the magnitude and phase of measured and predicted $S_{21}$. The magnitude of the model predictions was accurate to better than $2 \mathrm{~dB}$. The phase was accurate to better than 30 degrees overall, and better than 10 degrees between 4.8-5.5 GHz. A significant source of error is likely the absence of multiple scattering in the model and estimation errors from Eqn. (6). 


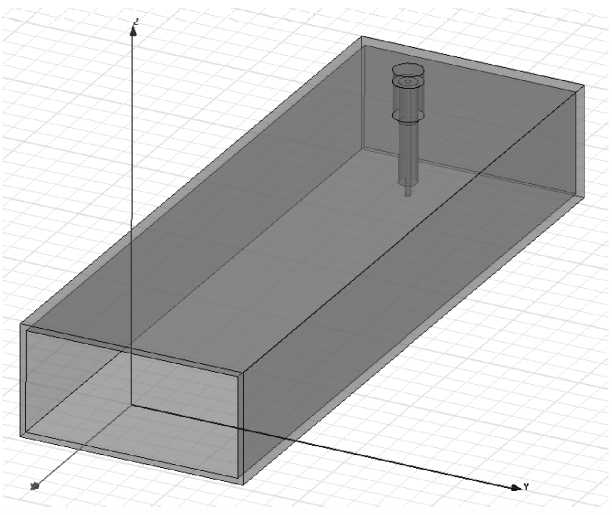

Figure 3: HFSS CAD model of probe-fed waveguide.

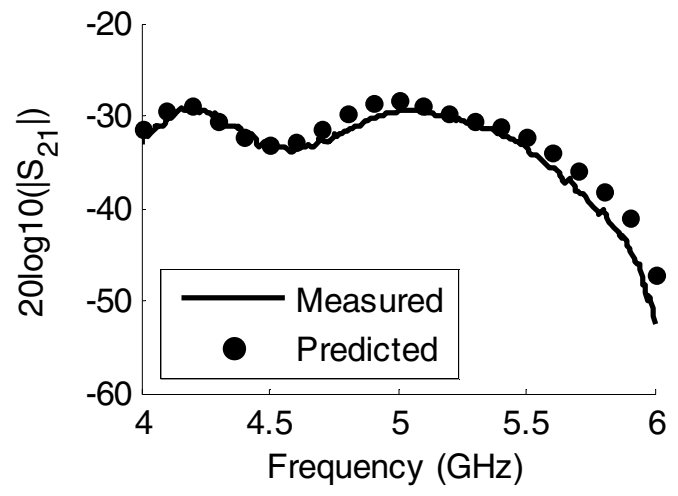

Figure 5: Magnitude of $S_{21}$, measured and predicted for the setup in Figure 4.

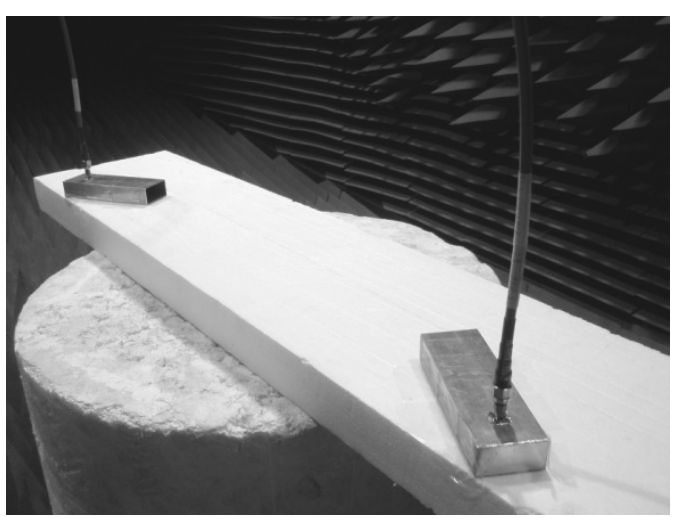

Figure 4: Anechoic chamber measurements of $S_{21}$. Waveguide apertures are separated by $0.55 \mathrm{~m}$ and rotated 30 degrees.

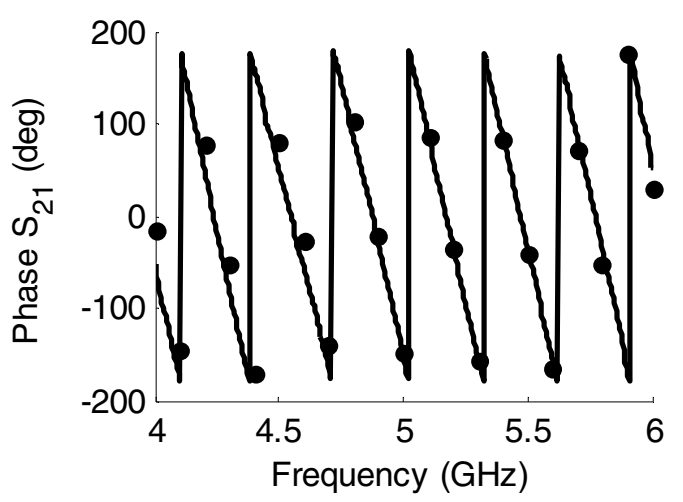

Figure 6: Phase of $S_{21}$, measured and predicted for the setup in Figure 4.

\section{Conclusion}

We have derived a model relating the multipole expansions of the fields of two antennas to S-parameter measurements between them. We also showed how numerical simulation can be used to approximately determine the antenna model parameters. We experimentally validated the model using two rectangular waveguides. Model predictions matched measurements to better than $2 \mathrm{~dB}$ in magnitude and better than 30 degrees in phase. In future work, we will investigate inverting Eqn. (5) directly for the transmit coefficients, as well as extend the model and experiments to include scattering from nearby objects.

\section{References}

[1] W. C. Chew. Waves and Fields in Inhomogeneous Media. New York: IEEE, 1995. [2] A.D. Yaghjian, "Scattering-Matrix Analysis of Linear Periodic Arrays," IEEE Trans. Antennas Propagat., vol. 50, pp. 1050-1064, August 2002 [3] R. A. Shore and A. D. Yaghjian, Scattering-Matrix Analysis of Linear Periodic Arrays of Short Electric Dipoles, Air Force Research Laboratory In-House Report, AFRL-SNHS-TR-2004-045, 2004.

[4] J. Hansen. Spherical Near-field Antenna Measurements. London: IEE Electromagnetic Waves Series 26, 1988. 